\title{
ns-level time transfer over a microwave link using the PTP-WR protocol
}

\author{
M. Rico, J.-P. Aubry, C. Botteron, P.-A- Farine \\ Ecole Polytechnique Fédérale de Lausanne, Electronics and Signal Processing Laboratory, \\ Neuchâtel, Switzerland \\ mathieu.rico@epfl.ch; cyril.botteron@epfl.ch; jean-pierre.aubry@epfl.ch; pierre-andre.farine@epfl.ch
}

\begin{abstract}
-today it is possible to achieve sub-ns level time synchronization on a wireline network while only us-level synchronization can be achieved on a wireless (microwave) link. In this paper we will, first, study the performances of different time synchronization wireline based protocols, such as Precision Time Protocol (PTP), Synchronous Ethernet (SyncE) and PTP White Rabbit (PTP-WR). And then, we will present our results using a wireless link, and determine which radio technology can achieve ns range time synchronization. Our motivation is to qualify a time transfer process operating over microwave link and offering secured GNSS-like time performance.
\end{abstract}

Keywords- microwave time transfer; PTP; IEEE1588; SyncE; PTP-WR,

\section{INTRODUCTION}

An increasing number of infrastructures require an accurate time reference, such as telecommunication base stations, energy industries (smart grid synchronization), and electronic intelligence systems. A GNSS receiver can provide an accurate time and frequency reference, but it requires a line-of-sight link to the satellites, and it has acknowledged vulnerabilities [1-4]. Therefore in some critical applications, a "GNSS-free" reference becomes mandatory [5].

Our goal is thus to identify a "GNSS-free" time transfer technology able to provide "some ns" level accuracy, traceable to UTC or to a private time reference, and to disseminate such secure and accurate time towards a fixed installation, ELINT or SIGNIT. The transport media should be wireless microwave on medium distance $(10-50 \mathrm{~km})$ to avoid fiber deployment within a given territory.

We have selected three time transfer protocols available on wireline: Precise Time Protocol (PTP); PTP with Synchronous Ethernet (PTP-SyncE); and PTP white rabbit (PTP-WR) [1014], which is an enhanced version of the PTP protocol [9] introduced by CERN providing time transfer in the sub-ns range over optical fiber links. Other physical approaches [7,15-16], involving GNSS based, SDH specific time stamp or amplitude modulation have been reported for time transfer. However they were not selected at that stage, because these techniques require specific HW/SW and do not seem ready for standardization. On the other hand, there is a significant amount of work dedicated to frequency accuracy and frequency stability transfer over fiber, mainly for primary clock comparison purpose [18-19]. These experiments are targeting the ultimate performance on frequency, and they can support dedicated HW/SW. Nevertheless, PTP-based protocols are preferred here because of equipment availability, Ethernet and IP network compatibility, and the launched standardization process of PTP-WR [17].

Currently, there is an increasing interest for PTP-WR over fiber, for short distance (less than $10 \mathrm{~km}$ ), as well as some very interesting developments on PTP-WR over long distance fiber [24]. However, while there exists some literature on PTP over wireless (see, e.g., [27]), there is not much published work yet on PTP-WR over wireless.

Therefore, our plan of work will be to confirm PTP-SyncE over wireless, PTP-WR over wireline, and then deploy PTP-WR over wireless, our final goal.

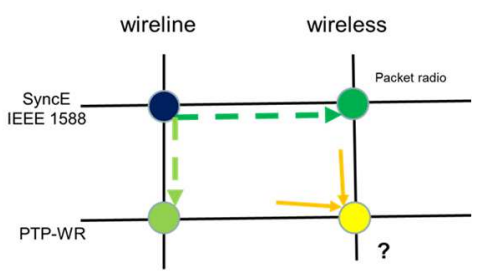

Fig. 1: evolution from PTP-WR over wireline and PTP over wireless towards PTP-WR over wireless

\section{A. Time transfer criteria}

Our purpose is to provide time transfer accuracy using, as much as we can, existing devices and protocols, already or close to be standardized.

The key criteria regarding time transfer functionality is accuracy, stability and precision (using wording definition of VIM, International Vocabulary for Metrology [6]). For the operational point of view, a time transfer process must be easy to calibrate and such calibration must be precise (in the sense of fidelity). Furthermore, that means that calibration must survive any switch off / switch on operation.

\section{B. PTP principle}

The PTP protocol, known as IEEE 1588, is an Ethernet based protocol, mostly introduced by Prof. Weibel [9], which is widely used in telecommunication and automation infrastructures. It can provide both time synchronization and frequency syntonization, and aims to provide sub-us level accuracy.

PTP protocol uses a "two-way time stamping" (similar as the one illustrated on the left of Fig. 2). Knowing those timestamps, the slave can identify its clock offset. The "two-way time

(C) 20xx IEEE. Personal use of this material is permitted. Permission from IEEE must be obtained for all other uses, in any current or future media, including reprinting/republishing his material for advertising or promotional purposes, creating new collective works, for resale or redistribution to servers or lists, or reuse of any copyrighted component of this work in other works. 
stamping" process is used also in other protocols such as NTP (Network Time Protocol), and PTP-WR. In PTP the propagation channel is assumed to be symmetrical, and time stamping can be done either in software or hardware (more accurate). PTP can be deployed on a classical IP network, but performance are highly improved by using PTP compliant intermediate devices such as Boundary Clock (BC) or Transparent Clock (TC) switches.

\section{SyncE concept}

Synchronous Ethernet is a standard that provides syntonization through an Ethernet network. It uses Ethernet idle patterns to encode a frequency reference on the physical layer. Specific hardware is needed to recover the frequency reference by using a PLL and all network devices must be SyncE compliant. Those constraints imply an upgrade of the whole network. It can achieve a $\pm 4.6 \mathrm{ppm}$ syntonization accuracy (ITU G.8262) (when the clock is not driven by an external reference) whereas standard Ethernet (IEEE 802.3) allows \pm $100 \mathrm{ppm}$.

Therefore, the syntonization process through SyncE might be highly efficient. SyncE is physically generated by $8 \mathrm{~B} / 10 \mathrm{~B}$ conversion (i.e., adding one bit every 4 bits), avoiding too many consecutive 1 or 0 's, and allowing to get a physical signal reference when data are processed and also when there is no data to convey. SyncE is a "master slave syntonization", like the good old time of SDH, while PTP is a two way process.

\section{White Rabbit presentation}

PTP-WR consists of three main aspects: it employs PTP to exchange timestamps and coarse synchronization; SyncE for syntonization; and a Dual Mixer Time Difference (DMTD) [10, 14]) for fine estimation synchronization. PTP-WR has a more accurate delay model which takes into account the hardware delay and the asymmetry of the propagation channel.

PTP-WR operation is summarized on Fig. 2:

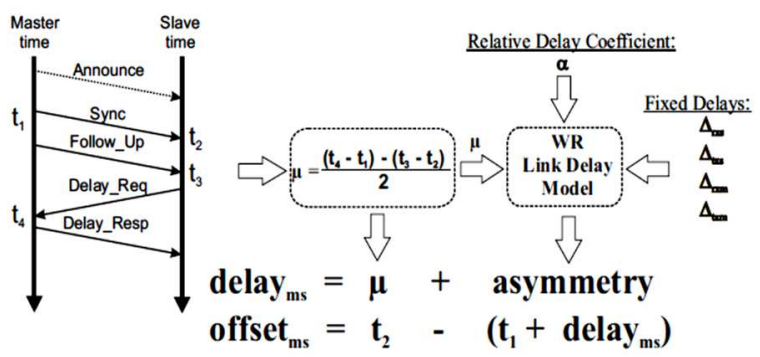

Fig. 2: PTP-WR operation [11]

A SyncE link is established forth and back, a first step synchronization using PTP is then performed (coarse acquisition) and a final step is applied (see Fig. 3), using the D.DMTD (Digital DMTD [14]) embedded in master and slave to perform a very accurate (sub wavelength) of the phase-time offset, by comparison between the phases of the outgoing optical signal and of the return signal (SyncE loop back).

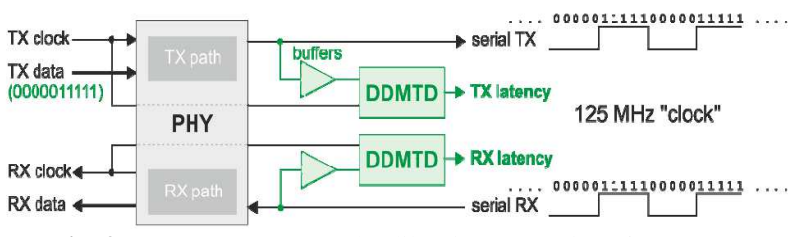

Fig. 3: PTP-WR SyncE and calibration extraction (from [11])

The PTP-WR delay link model, as define over wireline propagation, is defined on the following graph:

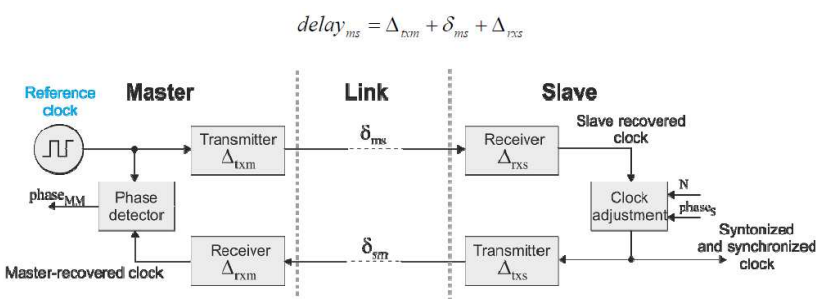

Fig. 4: PTP-WR delay link model (from [12])

Under wireless media, the "link" delay $\delta_{\mathrm{ms}}$ and $\delta_{\mathrm{sm}}$ will take into account the propagation delay and the time for media conversion interface $[18,19]$.

\section{E. Protocoles comparison}

PTP works only at the level of "clock" synchronization, time stamp, detecting the time of flight and assuming a ToF symmetry.

PTP-SyncE uses "one way syntonization", operating on a synchronous link, allowing a better time adjustment because of a common frequency reference.

PTP-WR uses a two way syntonisation, applies PTP "coarse" synchronization step, the two ways SyncE and the two D.DMTD allow to perform very accurate "phase detection".

\section{F. Goals and Methodology}

Our goal is to identify a time transfer technology adequate to disseminate some ns level accuracy over a wide area from a sync master to fixed equipement users.

Having selected an IP based generic solution, we will then compare the behavior of the various IP variants (PTP; PTPSyncE; PTP-WR) and we will also compare the behavior of each technology over various propagating media (wire, fiber, microwave).

A wireless IP packet radio introduces flight delay \& jitter, due to:

> Modulation scheme (X-PSK, X-QAM...), data rate (PTP is a 166 bytes process) due to latency and jitter in modulation / demodulation.

$>$ Frequency/Time selective channel.

$>$ Time or frequency spectrum allocation: FDD or TDD.

$>$ Half duplex / full duplex configuration.

(C) 20xx IEEE. Personal use of this material is permitted. Permission from IEEE must be obtained for all other uses, in any current or future media, including reprinting/republishing his material for advertising or promotional purposes, creating new collective works, for resale or redistribution to servers or lists, or reuse of any copyrighted component of this work in other works. 
$>$ Carrier frequency / bandwidth configuration.

\section{TIME TRANSFER OVER WIRELINE}

\section{A. PTP over wireline}

For comparison purpose, we have done some time transfer tests over wireline/fiber, using PTP, PTP-SyncE and PTP-WR.

The devices we used to qualify the PTP protocol are a PTP80 Grand Master Clock and PTP Slave from TIME \& FREQUENCY Solutions. The tests were done on a 100base-TX RJ45 8-meter copper cable after a transient phase of 2 hours to let the master stabilize its OCXO.

Fig. 5 shows the results we obtained with PTP over wireline.. On steady state, we can get an average pps (pulse per second) delay of $47 \mathrm{~ns}$ and a standard deviation of $3.7 \mathrm{~ns}$.

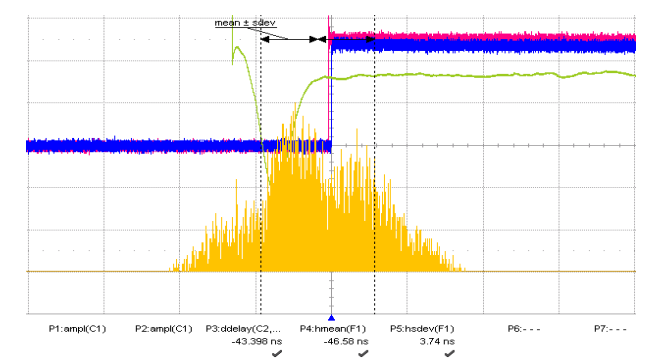

Fig.5. PTP protocol over 8-meter RJ45 cable: <delay> $47 \mathrm{~ns}-$ stdev $3.7 \mathrm{~ns}-$ red curve: master pps -blue: slave pps - green: pps delay - yellow: histogram

Those results seems to reach the lower bound of PTP performance, are well below the PTP protocol specification.

\section{B. PTP-SyncE over wireline}

PTP-SyncE was tested on a $5 \mathrm{~km}$ optic fiber. The test results are shown below:

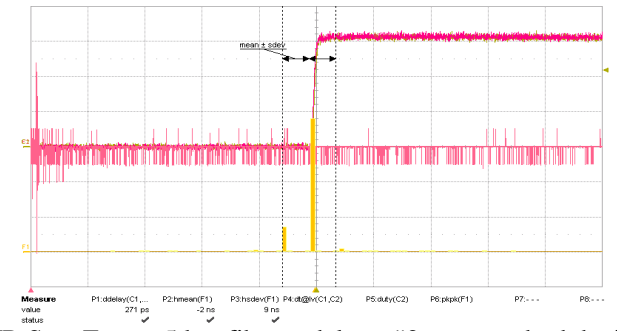

Fig. 6 : PTP-SyncE over 5 km fiber: <delay> \#2ns - standard deviation $9 \mathrm{~ns}$ red curve: master pps -brown: slave pps - pink: pps delay - yellow: histogram

SyncE over $5 \mathrm{~km}$ optical fiber (using optical SFP) shows a "bimodal" status with 2 lobes offset by around $10 \mathrm{~ns}$, which is close to one period of the SyncE carrier signal. The distribution is not Gaussian, and the "weighted average time offset" is about of $-2 \mathrm{~ns}$, while the dispersion ( $\mathrm{min} / \mathrm{max}$ range) is no more than $10 \mathrm{~ns}$.

Thus it seems that, at that stage, while PTP seems to be limited just below 100's ns range, the PTP-SyncE over fiber may reach some ns time transfer accuracy, and is able to exhibit a quite good stability.

Fig. 7 shows the initial time transfer series of Fig. 6. One can notice the initial transient time of the pps delay (in blue) oscillating within $+50 \mathrm{~ns}$ and $-60 \mathrm{~ns}$, and stabilizing after $200 \mathrm{~s}$.
After that, the oscillation remains within a $10 \mathrm{~ns}$ interval centered on $-2 n s$. This oscillation seems to be generated by $+/-$ 1 cycle of the SyncE carrier. This behavior could be a consequence of the hardware implementation of the PTP protocol on the PTP-SyncE master and slave devices we used.

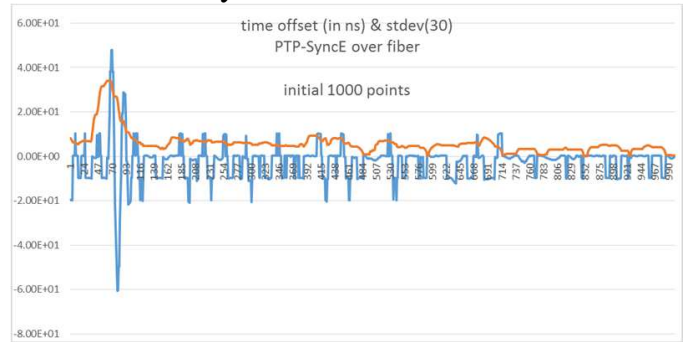

Fig. 7- PTP-SyncE time transfer over $5 \mathrm{~km}$ fiber: initial behavior

\section{PTP-WR over wireline}

The PTP-WR devices are supplied by 7 solutions, and consist in white rabbit switches and spec boards (PCI cards). We have performed two sets of characterization. One with a short fiber link (2m) and one using a $5 \mathrm{~km}$ long fiber. The following test results displayed on Fig. 8 were obtained by using PCI "spec boards" as master and slave. The distribution in each case is clearly Gaussian and the performance are well far under the sub-ns range, with an average delay of $120 \mathrm{ps}$ and a standard deviation of $20 \mathrm{ps}$.

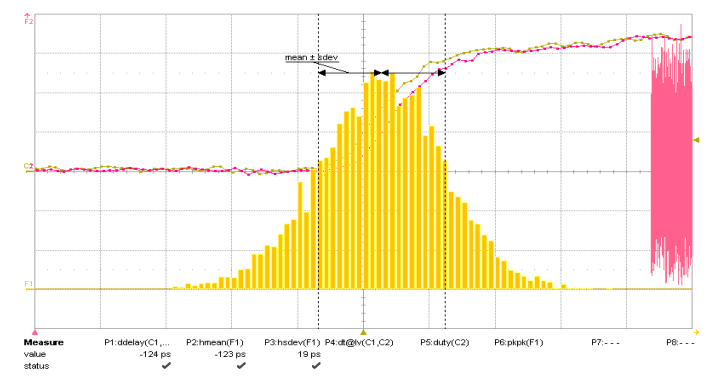

Fig.8. PTP-WR over $5 \mathrm{~km}$ optic fiber - <delay> 120 ps - stdev 20 ps red curve: master pps -brown: slave pps - pink: pps delay - yellow: histogram

\section{Time transfer noise analysis tools}

The Gaussian shape in Fig.8 shows that we are facing mostly white phase modulation (PM) noise, and this noise can be averaged out with integration time. The record of successive (\# 6000 points) pps offsets using WR over fiber is given in Fig. 9.

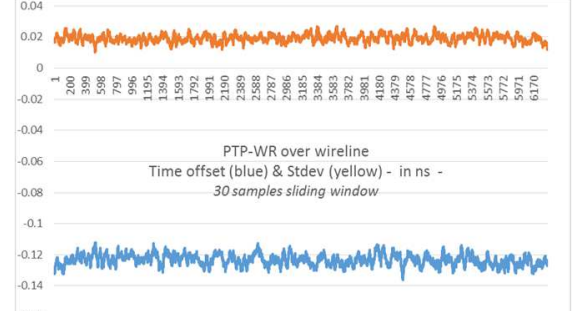

Fig. 9: PTP-WR over wireline - average and stdev - ns

Since late 90's, we know that the classical standard variance, $\sigma_{\mathrm{y}}{ }^{2}(\tau)=\left\langle(\mathrm{yi}-\langle\mathrm{yi}\rangle)^{2}\right\rangle$ is not applicable on clock signals because

(C) 20xx IEEE. Personal use of this material is permitted. Permission from IEEE must be obtained for all other uses, in any current or future media, including reprinting/republishing his material for advertising or promotional purposes, creating new collective works, for resale or redistribution to servers or lists, or reuse of any copyrighted component of this work in other works. 
of the environmental and inherent frequency drift, meaning that the average <yi > may drift with time, and the calculation will not converge with time and/or increasing number of samples. This is why D. Allan introduced the so called Allan variance [22], defined by the following equation:

$$
\sigma_{y}^{2}(\tau)=(1 / 2)\left\langle\left(y_{i+1}-y_{i}\right)^{2}\right\rangle
$$

The Allan variance removes drift and is able to converge under many conditions. Such analysis provides information on the shape of the noise contribution, and linear "asymptotic" fit in terms of $\sigma_{\mathrm{y}}^{2}(\tau)=\Sigma \mathrm{k}_{\mu} . \tau^{\mu}$ can be used to define the noise behavior. Allan variance is not able to isolate white PM from flicker phase modulation, and a modified Allan variance was later introduced [23]. When using time difference rather than relative frequency variation, one has to replace $y(t)$ in the previous equations by: $\mathrm{y}(\mathrm{k})=\left(\mathrm{x}_{\mathrm{k}+1}-\mathrm{x}_{\mathrm{k}}\right) / \tau, \tau$ being the equally spaced measurement time interval

In these formulas, we are using the classical definition of $\mathrm{x}(\mathrm{t})$, $\mathrm{y}(\mathrm{t})$ and $\phi(\mathrm{t})$, i.e. $\mathrm{x}(\mathrm{t})=\phi(\mathrm{t}) /\left(2 \pi \mathrm{v}_{0}\right), \mathrm{y}(\mathrm{t})=\left(1 /\left(2 \pi \mathrm{v}_{0}\right)\right) \mathrm{d} \phi(\mathrm{t}) / \mathrm{dt}$, and $y(t)=d x(t) / d t$. Overlapping samples was introduced to increase the time base of analysis despite a low number of samples.

When computing samples for any $\tau=m$. $\tau_{0}$ from time series collected every $\tau_{0}(1 \mathrm{~s})$ we get [23] an equivalent expression:

$$
\text { Mod. } \sigma_{y}^{2}(\tau)=\left(2 m^{2} \tau^{2}(N-3 m+1)\right)^{-1} \sum_{j=1}^{N-3 m+1}\left\{\sum_{i=j}^{j+m-1}\left(x_{i+2 m}-2 x_{i+m}+x_{i}\right)\right\}^{2}
$$

Samples are collected every seconds, and aggregate are calculated at overlapping $\tau=m \tau_{0}$ with $m=\{2,5,10,20 \ldots\}$. In Fig 10, the "Modified Allan variance vs time" $\sigma_{\mathrm{y}}{ }^{2}(\tau)$ of the time offset provided by PTP-WR over fiber exhibits a $\tau^{-3}$ slope. This is in agreement with similar WR over fiber analysis done by CERN team [26]. The time transfer process seems to be mainly affected by white noise PM.

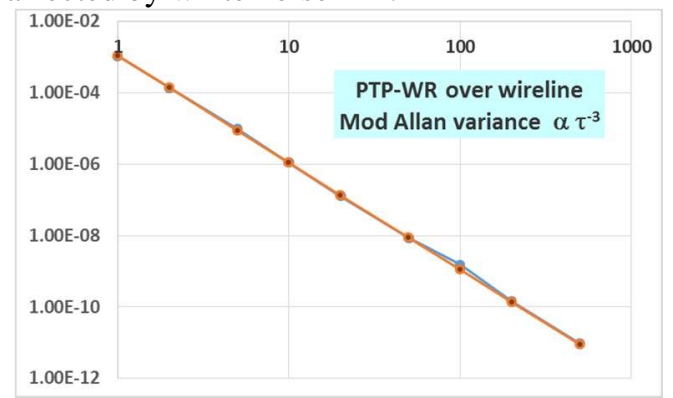

Fig. 10: Mod.Allan variance time offset: $\sigma_{\mathrm{y}}{ }^{2}(\tau)$ PTP-WR over wireline - blue curve: raw data - red: $\boldsymbol{\tau}^{-3}$ slope

This preliminary comparison between the various protocols and variants allowed us to qualify each protocol capability.

\section{TIME TRANSFER OVER WIRELESS}

As in the wireline case, we want to compare the time transfer behavior between the PTP variants (PTP, PTP-SyncE and PTP$\mathrm{WR}$ ) over various radio link configurations.

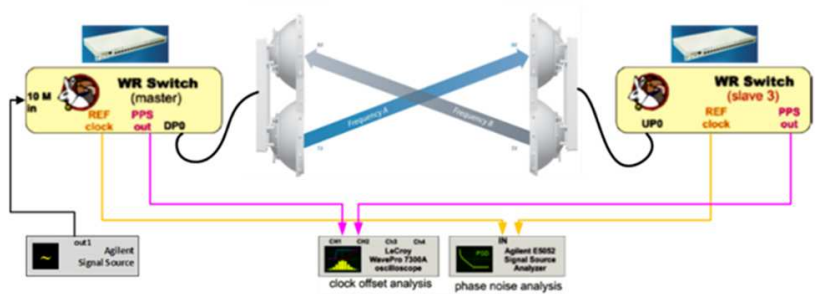

Fig. 11- Time transfer over wireless test configuration

The different radio link configurations are defined by the following parameters:

$>$ Modulation scheme and rate

$>$ FDD or TDD spectrum occupation

$>$ Carrier frequency and bandwidth

$>$ IP radio configuration: PTP compliant, PTP "transparent clock", one way SyncE, two way SyncE

The impact potentially generated by the microwave link might include some packet losses and a significant impact on Packet Delay Variation (PDV) at radio interface, coming from the conversion delay from wireline media to microwave, and queuing jitter.

\section{A. PTP over wireless}

To qualify PTP over wireless, we used the Cambium PtP 650 radio device [20], which provides several interesting features such as TDD (time duplex division), SyncE and PTP transparent clock feature (TC).

\section{1) Impact of the Transparent Clock feature}

With the TC feature disabled, we obtained when considering 40'000 pps delay measurements an average delay of 1.8 us and a standard deviation of $867 \mathrm{~ns}$, which is not within the PTP specifications. However, with the TC feature enabled (see Fig. 12), we obtained more interesting results: a time synchronization in the sub-100 ns level with an average delay of $50.5 \mathrm{~ns}$ and a standard deviation of $6.6 \mathrm{~ns}$. Note that a transient phase (see the circles on Fig.12) can also be observed. Such performance can be explained by the process employed by the PTP TC device. Actually, a TC device adds automatically residence time data to every PTP packet passing through, thus the PTP slave can take into account the jitter due to random queuing of the PTP packet in the network device.

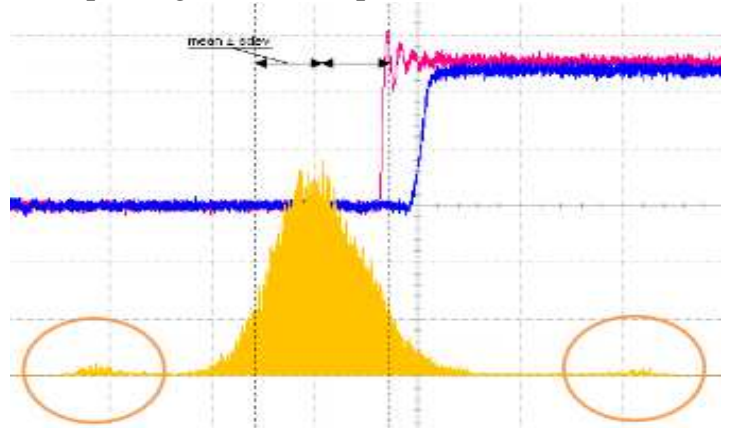

Fig.12. PTP over TDD - TC enabled: <delay> $50.5 \mathrm{~ns}-$ std dev $6.6 \mathrm{~ns}$ red curve: master pps -blue: slave pps - green: pps delay - yellow: histogram

(C) 20xx IEEE. Personal use of this material is permitted. Permission from IEEE must be obtained for all other uses, in any current or future media, including reprinting/republishing his material for advertising or promotional purposes, creating new collective works, for resale or redistribution to servers or lists, or reuse of any copyrighted component of this work in other works. 


\section{2) Modulation impact BPSK vs.QAM}

The microwave link used previously can be adapted from 256QAM (previous configuration) to BPSK. The observed performance with PTP, no SyncE, TC, is provided in Fig. 13:

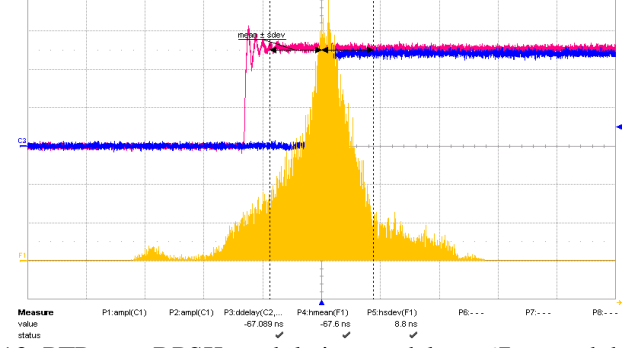

Fig. 13: PTP over BPSK modulation - <delay > 67ns - std dev 9ns

From this figure, we measured with BPSK modulation a mean offset of $-38 \mathrm{~ns}$ and a standard deviation of $9 \mathrm{~ns}$, very similar as under 256QAM configuration (for which the average delay was $50.5 \mathrm{~ns}$ and a standard deviation of $6.6 \mathrm{~ns}$ ).

\section{3) FDD versus $T D D$}

The AirFiber module from Ubiquity allows us to configure the access technique either in FDD or TDD. In both conditions we observed a transient initial drift "time to set" greater than $1 \mu \mathrm{s}$, up to a thousand seconds, and a steady state offset and fluctuations of some hundreds of ns.

At that stage, we can conclude that PTP over wireless may allow a time sync less than $100 \mathrm{~ns}$ with a dispersion below 10 ns, but only with the TC enabled configuration. No significant impact of radio link configuration (FDD/TDD, BW, modulation, data rate...) is observed under PTP operation.

\section{B. PTP-SyncE over wireless}

We have performed time offset measurement based on PTPSyncE over a FDD microwave link provided by Bridgewave [21].

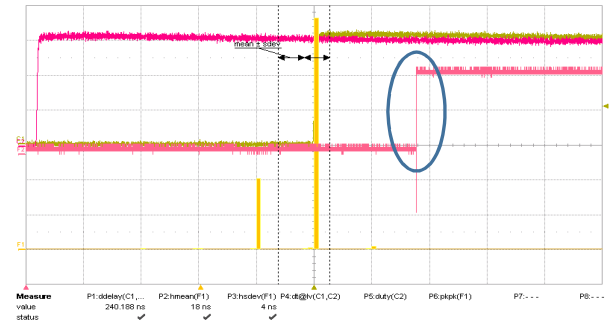

Fig. 14: PTP SyncE over FDD - average delay 18ns - std dev 4.5 ns (calculated on the first 135000 points)

The 240 ns pps delay jump (circled in Fig. 14) at $135^{\prime} 000^{\text {th }}$ event is not yet understood. Domains before and after the delay jump, present a "Gaussian" population with a standard deviation below $5 \mathrm{~ns}$.

On each domain the time transfer offset exhibits two stable states separated by $10 \mathrm{~ns}$ (shown on the yellow histogram).
We have analyzed the 40'000 initial points along with Mod.Allan variance.

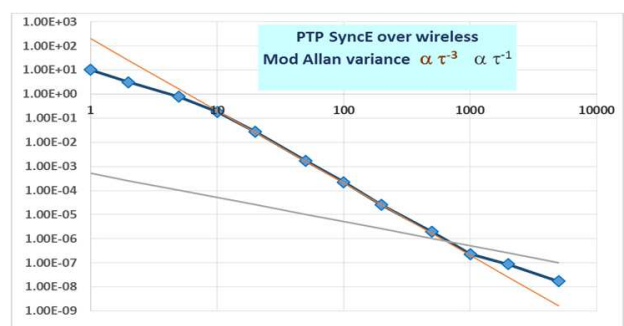

Fig. 15: PTP-SyncE over FDD - pps offset: $\operatorname{Mod} . \sigma_{y}(\tau)^{2}$ plot

In Fig 15 , the Mod. $\sigma_{\mathrm{y}}(\tau)^{2}$ plot shows a $\tau^{-3}$ slope between 10 and 1000 s, indicating a white phase modulation contribution, and behaves as $\tau^{-1}$ (or $\tau^{-1.5}$ ) below 10s and higher than $1000 \mathrm{~s}$.

\section{PTP-WR over wireless}

As previously discussed, PTP-WR uses PTP to perform a raw estimate of time offset, and use DMTD to determine fine time offset, by measuring the phase on the optical link from master to slave and from slave to master. This is the reason why we need to have a "forth \& back" SyncE compliant radio link, and a very stable propagation delay. Therefore, PTP-WR synchronization has not yet been obtained on wireless, because the propagation delay (calculated by using the timestamp exchanged) introduced by the radio is too jittery, as we can see on Table 1 that presents the calculated propagation delay on fiber and wireless link, using the white rabbit timestamps, $\mathrm{t} 1, . ., \mathrm{t} 4$ which are defined on Fig. 2 .

Table 1 : propagation delay calculated with timestamps

\begin{tabular}{|c|c|c|c|c|}
\hline & Average t2-t1 & STD dev t2-t1 & Average t4-t3 & STD dev t4-t3 \\
\hline 2m fiber & 0.3 us & $3.4 \mathrm{ps}$ & 0.2 us & $4.0 \mathrm{ps}$ \\
& & 1.6 us & 25.5 us & $1.6 \mathrm{us}$ \\
\hline Wireless & 38.4 us & & & \\
& & &
\end{tabular}

We have collected approximately 1300 timestamps exchanges between the PTP-WR master and slave, and the calculation shows that the delay variation and standard deviation due to the radio are significantly larger than in the wireline situation.

To overcome the packet delay variation issue, we are planning to design our own radio interface. It will have the following characteristics:

- FDD access technique to avoid the complexity of TDD radio synchronization

- Bidirectional SyncE feature (mandatory for delay estimation).

- $\quad$ IP packet queuing with fixed delay to avoid jitter.

It must be noted that the latency specified in the FDD link we are working with (see [23]) is given as less than $30 \mu$ s, which would be equivalent to a time of flight of $10 \mathrm{~km}$ over fiber. Also FDD in the microwave technique is the equivalent of the WDM (Wavelength Division Multiplexing) in optic fiber domain.

(C) 20xx IEEE. Personal use of this material is permitted. Permission from IEEE must be obtained for all other uses, in any current or future media, including reprinting/republishing his material for advertising or promotional purposes, creating new collective works, for resale or redistribution to servers or lists, or reuse of any copyrighted component of this work in other works. 


\section{DISCUSSION}

We have shown that accurate and stable time transfer may be obtained through proper wireless link.

We have identified the contribution of SyncE vs basic PTP, and the advantage of PTP-WR vs. PTP-SyncE. The synchronization accuracy is highly dependent to the Master and Slave PTP protocol implementation. Proper radio link can be specified as PTP compliant (supporting TC feature [25]), and SyncE.

The impact of modulation (256QAM, BPSK) and various bandwidth does not reveal a major impact at PTP level. This should be updated under PTP-WR configuration, expected to be more sensitive. So far, we have not made any attempt to control or modify calibration figures in the link delay model.

\section{PRELIMINARY CONCLUSION}

Our aim is to qualify a "GNSS-free" time transfer solution, allowing microsecond and sub microsecond accuracy.

We have realized time transfer over various protocols (PTP, PTP-SyncE and PTP-WR), and we have tested various microwave link configurations.

So far we have been able to get time transfer accuracy under $50 \mathrm{~ns}(1 \sigma 10 \mathrm{~ns})$ based on PTP-SyncE over low jitter FDD and TDD radio links, under the condition of a "transparent clock" PTP behavior. This is still far away from PTP-WR or PTPSyncE performance over optical fiber, as shown on fig 8 .

At the accuracy of $50 \mathrm{~ns}$, we have not seen any significant impact of TDD/FDD, spectrum allocation, radio modulation, or bandwidth. SyncE has a major impact on time transfer performance, both on accuracy or on stability.

We have been able to quantify the basic requirements of a PTP-WR microwave radio link. Next steps will be to define an HW (FPGA) radio interface between PTP-WR devices and a dedicated microwave link.

\section{ACKNOWLEDGMENTS}

We warmly thank people and companies who have provided us hardware and support. Time\&Frequency Solution-T\&FS (UK) providing the PTP-80 master/slave devices, and Meinberg (G) providing the PTP-SyncE master / slave devices. The PTPWR are from 7Solutions. The main wireless results were obtained over Cambium Networks and Bridgewave radio link. Thanks to all of them for their support.

We warmly thank our sponsor, Armasuisse, under the contract 8003505738 ARAMIS-No. 041-21 for funding this project in EPFL ESPLAB.

\section{REFERENCES.}

[1] J.A. Volpe, "Vulnerability Assessment of the transportation infrastructure relying on the Global Positioning System", In National Transportation Systems Center, 2001

[2] A. Jafarnia-Jahromi, A. Broumandan, J. Nielsen, and G. Lachapelle, "GPS vulnerability to spoofing threats and a review of antispoofing techniques", In International Journal of Navigation and Observation ,2012

[3] P. Papadimitratos \& A. Jovanovic, "Protection and Fundamental Vulnerability of GNSS", In IEEE International Workshop on Satellite and Space Communications, IWSSC, 2008.

[4] R.H. Mitch, et al., "Civilian GPS Jammer Signal Tracking and Geolocation", In Proceedings of Institute of Navigation GNSS, 2012.

[5] J-P. Aubry, "Precise time generation and dissemination in power networks: available technologies and trends to address Smart Grid needs", Electromagnetics in Renewable Energies, SEREC, Swiss Electromagnetics Research \& Engineering Center, 2012,

[6] International Vocabulary of Metrology - Basic and general concepts and associated terms (VIM), $3^{\text {rd }}$ edition, JCGM,2012

[7] J.Levine, "A review of time and frequency transfer methods", Metrologia 45 (2008) S162-S174

[8] R. Merz, "Analysis of Low Power, Low Data Rate Ultra Wideband Impulse Radio Systems", PhD Thesis, Université de Neuchâtel Institut de Microtechnique, 2009

[9] H. Weibel, "IEEE 1588 Tutorial," In 2006 Conference on IEEE 1588. Oct 1, 2006, NIST, Gaithersburg, NISTIR.

[10] G. Daniluk "White Rabbit PTP Core - the sub-nanosecond time synchronization", Master of Science thesis, Warsaw, 2012

[11] T. Włostowski, "Precise time and frequency transfer in a White Rabbit network", Master of Science thesis, 2011

[12] M. Lipinski, "White Rabbit: Ethernet-based solution for sub-ns synchronization and deterministic, reliable data delivery", IEEE Plenary Meeting Geneva, 15 July 2013

[13] A.A.Söderqvist, N.Claesson:A Timing System Application using White Rabbit, Master Thesis, LUND University, 2014.

[14] P. Moreira, I. Darwazeh, "Digital femtosecond time difference circuit for CERN's timing system", August 2011, online: www.ee.ucl.ac.uk/lcs/previous/LCS2011/LCS1136.pdf

[15] P.O. Hedekvist, S.C. Ebenhag, "Time and Frequency Transfer in Optical Fibers", Recent Progress in Optical Fiber Research, ISBN 978953-307-823-6, 2012

[16] O. Lopez, \&al. , "Simultaneous remote transfer of accurate timing and optical frequency over a public fiber network", Springer Journal of Applied Physics B, 2012.

[17] M. Lipinski, White Rabbit Standardisation in IEEE 1588 (PTP), White Rabbit Worshop, CERN, Octobre 2014

[18] R. Exel, T. Bigler, and N., Kero, "Asymmetry Mitigation through Line Swapping in IEEE 802.3 Ethernet", in Proceedings of Precise Time and Time Interval Systems and Applications Meeting (PTTI'2012), Reston.

[19] A. Rahman, PhD Thesis, "Delay Asymmetry Correction Model for IEEE 1588 Synchronization Protocol", presented at Electrical and Computer Engineering, Carleton University Ottawa, Ontario, 2012.

[20] Cambium Network : PTP 650 SPECIFICATION SHEET, 2014

[21] BridgewaveFlexPort® A Better Approach to High-Capacity Microwave Backhaul, White paper, 2014; data sheet AR80 \& AR80X PtP, 2014

[22] D.Allan, M.Weiss \& J.Jespersen, "A Frequency-domain view of timedomain characterisation of clocks and time \& frequency distribution systems", 45 ${ }^{\text {th }}$ IEEE-IFCS, 1991

[23] WJ Riley, NIST Special Publication 1065 - Handbook of Frequency Stability Analysis, 2008

[24] E. Dierikx, T. Pinkert, J. Koelemeij, H. Peek, Rob Smets, Using "White Rabbit PTP for Accurate Time and Frequency Transfer in Long Haul Optical Fiber Links", these proceedings, 2015

[25] C.Prados, “White Rabbit Transparent Clock”, IEEE ISPCS 2013

[26] M. Lipinski \& al. "Performance results of the first White Rabbit installation for CNGS time transfer", IEEE ISPCS 2012

[27] C.N.M Marins \& al. "Precision Clock and Time Transfer on aWireless Telecommunication Link", IEEE TRANSACTIONS ON INSTRUMENTATION AND MEASUREMENT, VOL. 59, NO. 3, MARCH 2010

(C) 20xx IEEE. Personal use of this material is permitted. Permission from IEEE must be obtained for all other uses, in any current or future media, including reprinting/republishing his material for advertising or promotional purposes, creating new collective works, for resale or redistribution to servers or lists, or reuse of any copyrighted component of this work in other works. 